\title{
Pulmonary vascular resistance in repaired congenital diaphragmatic hernia vs. age-matched controls
}

\author{
Matthew E. Zussman', Michelle Bagby', D. Woodrow Benson', Resmi Gupta' and Russel Hirsch'
}

INTRODUCTION: Infants and children with repaired congenital diaphragmatic hernia (CDH) often continue to show delayed growth and development that may be, in part, secondary to unrecognized persistence of increased pulmonary vascular resistance (PVR).

METHODS: Data were reviewed from all patients ages 6-36 mo with repaired $\mathrm{CDH}$ who underwent cardiac catheterization from 2007 to 2010 and were compared to data from a control population of patients undergoing percutaneous closure of a patent ductus arteriosus (PDA). Indexed pulmonary blood flow (Qp), mean pulmonary artery pressure (mPAP), pulmonary capillary wedge pressure (PCWP), and PVR were examined.

RESULTS: Data from $8 \mathrm{CDH}$ patients and 10 control patients were examined. The mPAP $(22.5 \pm 3.33$ vs. $18.2 \pm 4.13 \mathrm{~mm}$ $\mathrm{Hg})$ and PVR (3.66 \pm 0.79 vs. $1.22 \pm 0.4 \mathrm{iwU}$ (indexed Wood's units)) were both significantly elevated in the $\mathrm{CDH}$ population, whereas the Qp ( $4.08 \pm 1.43$ vs. $\left.6.82 \pm 1.46 \mathrm{l} / \mathrm{min} / \mathrm{m}^{2}\right)$ was significantly lower in this population. There was no significant difference in pulmonary capillary wedge pressure (PCWP). Less than half of the $\mathrm{CDH}$ patients had signs of pulmonary hypertension (PH) on echocardiogram.

DISCUSSION: Our data suggest that children who are ages 6-36 mo with repaired CDH have significantly increased PVR compared with controls and early consideration of cardiac catheterization may be warranted.

C ongenital diaphragmatic hernia $(\mathrm{CDH})$ has an incidence of approximately 1 in 3,000 births and is caused by a defect in the fusion of the pleuroperitoneal membranes during development (1-3). Although the etiology of this disease is poorly understood, both genetic and environmental factors are thought to play a role $(4,5)$. Prior to surgical repair, mortality is directly related to the level of pulmonary hypertension $(\mathrm{PH})(6)$. This has led to the development of different strategies aimed at relieving elevation of pulmonary artery $(\mathrm{PA})$ pressures in the preoperative period, with a resulting improvement in survival.

Most patients who survive to hospital discharge have some level of normalization of PA pressures as estimated by echocardiography. Also, respiratory reserve appears to improve significantly in the first years of life, with less than $2 \%$ of these children requiring oxygen at $2 \mathrm{y}$ of age (7). However, there is recent evidence that there is a much higher incidence of respiratory tract infections and failure to thrive in these children $(8,9)$. Although very little is known regarding the pulmonary vascular outcomes in these patients, electrocardiographic data have shown that nearly $40 \%$ of survivors near 3 y of age meet criteria for $\mathrm{PH}$ with right-axis deviation or right ventricular hypertrophy $(10,11)$. To date, no systematic invasive hemodynamic study exists in this patient population.

In this study, we report the hemodynamic findings in patients with repaired $\mathrm{CDH}$ obtained during clinically indicated cardiac catheterization compared to an age-matched control population undergoing elective closure of a small patent ductus arteriosus (PDA).

\section{RESULTS}

Characteristics of $\mathrm{CDH}$ and Control Subjects

A total of 8 study patients (mean age $16.9 \pm 9.3$ mo; mean weight $8.7 \pm 2.3 \mathrm{~kg}$ ) and 10 control patients (mean age $17.3 \pm 8.0 \mathrm{mo}$; mean weight $10.7 \pm 2.6 \mathrm{~kg}$ ) were identified. There was no difference in the demographics between the two groups (Table 1).

The average gestational age was $37 \mathrm{wk}$ in both the $\mathrm{CDH}$ (range 35-39) and the control (range 31-41) populations. The birth weight was $3.07 \mathrm{~kg}$ (range 2.28-3.50) and $2.90 \mathrm{~kg}$ (range $1.53-4.15)$ in the $\mathrm{CDH}$ and control groups, respectively. The mean age of $\mathrm{CDH}$ repair was $25 \mathrm{~d}$ (range 6-45). Most hernias (75\%) were left sided. Three patients $(37.5 \%)$ required extracorporeal membrane oxygenation support during initial hospitalization. The average length of hospital stay for the $\mathrm{CDH}$ group was $153.5 \mathrm{~d}$, with a median stay of $135 \mathrm{~d}$. Based on review of medical records, the indications for catheterization in the study group included abnormal echocardiograms (three patients), growth delay of unknown cause (six patients), and/ or recurrent respiratory symptoms (three patients). Five of the eight $\mathrm{CDH}$ patients were taking sildenafil and one was on home oxygen at the time of catheterization.

\section{Characteristics of Echocardiograms}

All sedated echocardiograms were performed less than 2 mo prior to cardiac catheterization. Three of the $\mathrm{CDH}$ patients (37.5\%) and one of the control patients had signs of $\mathrm{PH}$ on their echocardiogram (tricuspid regurgitation or PDA gradient estimating right-sided pressure $>40 \%$ systemic pressure). 
Table 1. Baseline demographic data of the study $(\mathrm{CDH})$ and control (PDA) populations

\begin{tabular}{lcc}
\hline & CDH & PDA \\
\hline Patients & 8 & 10 \\
Sex, male \% & $62.5 \%$ & $40 \%$ \\
Mean GA (wk) & $37.3 \pm 1.8$ & $37.3 \pm 4.3^{\mathrm{a}}$ \\
Mean birth weight (kg) & $3.07 \pm 0.45$ & $2.90 \pm 1.04^{\mathrm{a}}$ \\
Mean weight at cathetherization (kg) & $8.7 \pm 2.3$ & $10.7 \pm 2.6$ \\
Mean age at catheterization (mo) & $16.9 \pm 9.3$ & $17.3 \pm 7.97$ \\
Hernia side, left & $6(75 \%)$ & $\mathrm{N} / \mathrm{A}$ \\
Need for ECMO & $3(37.5 \%)$ & $\mathrm{N} / \mathrm{A}$ \\
Mean age at CDH repair (d) & $25.6 \pm 14.1$ & $\mathrm{~N} / \mathrm{A}$ \\
\hline
\end{tabular}

$\mathrm{CDH}$, congenital diaphragmatic hernia; ECMO, extracorporeal membrane oxygenation; GA, gestational age; N/A, not applicable; PDA, patent ductus arteriosus.

${ }^{a} \mathrm{G} A$ and birth weight data available for 8/10 PDA patients.

\section{Cardiac Catheterization Results}

Analysis of the catheterization data shows that the mean pulmonary artery pressure (mPAP) $(22.5 \pm 3.33$ vs. $18.2 \pm 4.13 \mathrm{~mm}$ $\mathrm{Hg}, P<0.01)$ and pulmonary vascular resistance (PVR) $(3.66 \pm$ 0.79 vs. $1.22 \pm 0.4$ indexed Wood's units (iwU), $P<0.01$ ) were both significantly higher, and the Qp (4.08 \pm 1.43 vs. $6.82 \pm$ $1.46 \mathrm{l} / \mathrm{min} / \mathrm{m}^{2}, P=0.03$ ) significantly lower, in the $\mathrm{CDH}$ group compared with the controls. The pulmonary capillary wedge pressure (PCWP) was slightly lower in the study patients (7.81 \pm 2.7 vs. $9.45 \pm 2.7 \mathrm{~mm} \mathrm{Hg}, P=0.24)$ but did not reach statistical significance (Figure 1).

\section{Echocardiographic vs. Cardiac Catheterization Assessment of PH} The average PVR of the $\mathrm{CDH}$ patients who had abnormal precatheterization echocardiograms was 3.75 iwU (individually $3.05,4.03,4.17)$ compared with the remainder of the study patients, for whom the average PVR was $3.62 \mathrm{iwU}$. The PVR of the one control patient with abnormal echocardiogram was $1.0 \mathrm{iwU}$.

\section{DISCUSSION}

There have been significant strides in the initial care of patients born with $\mathrm{CDH}$, and considerable attention is now routinely given to the evaluation and treatment of $\mathrm{PH}$ during the preand perioperative period. Consequently, there has been a significant improvement in short-term outcomes. With this increased early survival, consideration can now be given to $\mathrm{PH}$ risk and long-term pulmonary vascular prognosis.

This report compares repaired $\mathrm{CDH}$ patients with a control population and provides the first insights into the pulmonary vascular hemodynamic outcome after repair of this defect. The major finding of this study was that invasive hemodynamics in the repaired $\mathrm{CDH}$ population showed elevated baseline PA pressures and PVR compared with age-matched controls. The elevated PA pressures were noted to be independent of elevation of PCWP. In addition, we unexpectedly found that pulmonary blood flow is diminished in the $\mathrm{CDH}$ patients we studied. To the best of our knowledge, these are the first comparative invasive hemodynamic data reported in this survivor population.
The etiology of $\mathrm{PH}$ in this population is complex. Histological studies of lung parenchyma in these patients have shown a reduced total pulmonary vascular bed with decreased numbers of vessels per unit lung. Medial hyperplasia of the pulmonary arteries together with peripheral extension of the muscle layer into the small arterioles has also been noted (12-16). Further, in the CDH lung, there is failure of arterial wall thinning associated with normal newborn pulmonary arterial remodeling (14). Despite these pathologic changes, clinical respiratory reserve appears to improve significantly in the first years of life, with very few survivors requiring oxygen in early childhood (7). There are, however, conflicting noninvasive data showing that nearly half of early childhood survivors have signs of $\mathrm{PH}$ (11).

Elevation of pulmonary artery pressure in the face of lownormal pulmonary blood flow would suggest the presence of pulmonary vasoconstriction and/or a diminution of total cross-sectional vascular area. The chronic exposure of the right ventricle to higher resistance may account for the subclinical ventricular dysfunction and the lower-than-expected pulmonary blood flow that was documented. Given the expectation that further pulmonary growth and parenchymal maturation may continue later into childhood in these survivors, therapies to treat pulmonary vascular disease and preserve cardiac function in the interim may be vital.

The $\mathrm{CDH}$ patients evaluated in this report were electively studied at times of clinical indication, and only a single point of hemodynamic data was obtained. Further, a clear selection bias exists in that the study included only those subjects who met minimum clinical criteria to undergo cardiac catheterization. Thus, these preliminary data cannot be extrapolated to the general $\mathrm{CDH}$ population at this time. Presumably, the hemodynamic findings in the $\mathrm{CDH}$ population as a whole would fall in a spectrum extending from normal to grossly abnormal, and only a prospective clinical protocol that includes elective cardiac catheterization would help determine their exact course. The impact of this bias may be blunted by the lack of overt evidence of $\mathrm{PH}$ on echocardiogram in a large percentage of patients undergoing catheterization. The $\mathrm{CDH}$ patients evaluated in this study had clinical findings significant enough for the physician to recommend cardiac catheterization; those catheterizations subsequently provided the first concrete evidence of $\mathrm{PH}$.

Although it was not the purpose of the study to compare the assessment of $\mathrm{PH}$ by catheterization vs. echocardiogram, and our study was underpowered for this question, we found that there was no obvious difference in the average PVR in those with abnormal precatheterization echocardiograms compared with those who had other indications for catheterization. This emphasizes the importance of obtaining invasive hemodynamic data in repaired $\mathrm{CDH}$ patients who are clinically concerning.

In addition to those discussed above, the conclusions derived from this retrospective chart review carry other limitations and assumptions. Unfortunately, little normative data exist regarding invasive hemodynamics in this age population, and the selected PDA population is the most reasonable for use as a control. If this population has a higher PVR compared 

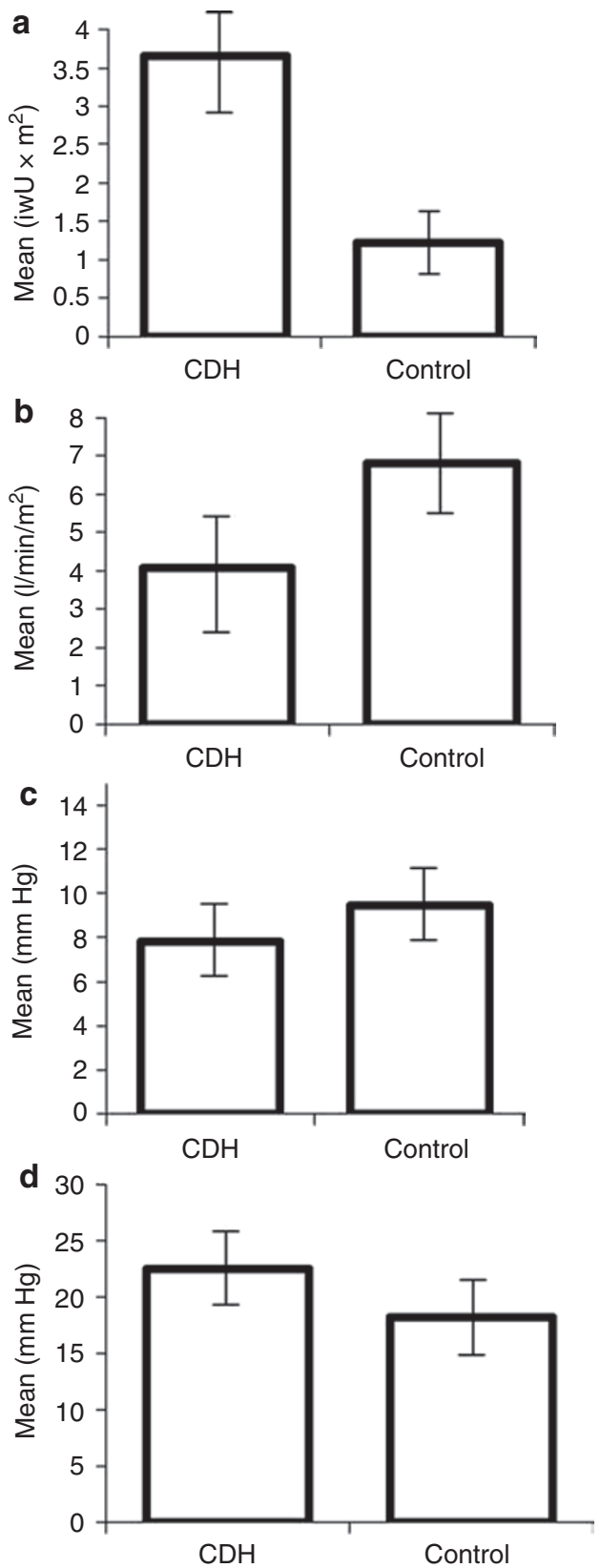

Figure 1. Comparison of invasive hemodynamic data between the study $(\mathrm{CDH})$ and control (PDA) populations. All panels display the mean with bars of standard deviation. Panels show (a) PVR, (b) Qp, (c) PCWP, and (d) mPAP. CDH, congenital diaphragmatic hernia; mPAP, mean pulmonary artery pressure; PCWP, pulmonary capillary wedge pressure; PDA, patent ductus arteriosus; PVR, pulmonary vascular resistance; Qp, indexed pulmonary blood flow; iwU, indexed Wood's units.

with a true control population due to prolonged left-to-right shunt through their PDA, the results we have presented may be biased toward the null (type II error).

In 2008, the American Academy of Pediatrics (AAP) Section on Surgery and the Committee on Fetus and Newborn released their recommended follow-up schedule for infants with $\mathrm{CDH}$. This guideline included post-discharge echocardiography only if the patient is on oxygen or previously had an abnormal echocardiogram (17). Our results suggest that serial echocardiography may be of limited utility because this modality may substantially underestimate the extent of $\mathrm{PH}$ and in no way can help determine PVR or pulmonary blood flow. This finding is consistent with other studies that have shown that echocardiography for assessment of $\mathrm{PH}$ in the pediatric population may have variable utility (18). We suggest that closer follow-up, possibly including cardiac catheterization, may be necessary in any patient who deviates from the expected clinical course. All infants born with $\mathrm{CDH}$ should follow the AAP guidelines for preventive pediatric health for high-risk infants.

In conclusion, this study provides concerning preliminary data and serves as a foundation for further investigation. Systematic prospective evaluation is necessary to enhance our understanding of the long-term outcome of $\mathrm{PH}$ after $\mathrm{CDH}$ repair and could ultimately guide therapeutic intervention.

\section{METHODS}

Following approval from the institutional review board at Cincinnati Children's Hospital Medical Center, a search of a divisional database for all patients aged 6-36 mo with repaired $\mathrm{CDH}$ who had undergone cardiac catheterization between 1 January 2007 and 1 January 2010 was performed. The subjects were excluded if there were significant comorbidities that may independently elevate PVR (including known genetic disorder or congenital heart disease, e.g., large, unrepaired intracardiac shunts). Patients were also excluded if an accurate PVR could not be calculated. Patients with small atrial septal defects, repaired/resolved PDA, or ventricular septal defects were included in the study.

Control subjects included patients in the same age range undergoing cardiac catheterization for PDA closure in which a complete hemodynamic data set was present after PDA closure. The control population was chosen on the basis of the absence of chronic lung disease (as determined by history and chest radiograph) and normal PVR. As a group, they represent the only patients undergoing cardiac catheterization with isolated extra-cardiac defects and no intracardiac abnormalities. In addition to the same exclusion criteria applied to the study subjects, controls were excluded if the initial (pre-PDA closure) indexed pulmonary blood flow (Qp):indexed systemic blood flow (Qs) ratio was greater than 2:1. This criterion was applied in order to avoid control subjects with significant left ventricular volume load, which can cause diastolic dysfunction and secondary elevation of pulmonary artery pressure. The control patients were identified from the same divisional database as the study subjects.

In addition to baseline demographic and gestational data, the following hemodynamic variables were collected for comparison between the study and control groups: Qp, mPAP, PCWP, and PVR. If patients had more than one cardiac catheterization, the most recent study data prior to age 36 mo were evaluated.

For all included patients, the most recent sedated echocardiogram was examined for signs of $\mathrm{PH}$, including right ventricular pressure (by estimate of tricuspid valve doppler flow velocity), PA diastolic pressure (by estimate of pulmonary regurgitation doppler flow velocity), or gradient across a PDA if present (by estimate of doppler flow gradient between the aorta and PA). In addition, any septal flattening as seen in the parasternal short-axis view apical to the level of the mitral valve was also noted. The results of $\mathrm{CDH}$ patients with abnormal echocardiograms were compared with the results of those with normal findings.

Basic descriptive statistics were used to compare demographic variables. Further analysis of data, including mean, median, standard deviation, minimum, maximum, and interquartile range, was performed for each variable (Table 1). Because of the small sample size, the exact Wilcoxon two-sample test was used to compare the differences in Qp, mPAP, PCWP, and PVR between the CDH and control groups. The criterion for statistical significance was set at the nominal $a=0.05$ level. All analyses were conducted using commercially available software (SAS version 9.2; SAS Institute, Cary, NC). 


\section{Articles $\mid$ Zussman et al.}

\section{STATEMENT OF FINANCIAL SUPPORT}

This study was funded internally.

Disclosure: The authors have no conflict of interest to disclose.

\section{REFERENCES}

1. Skarsgard ED, Harrison MR. Congenital diaphragmatic hernia: the surgeon's perspective. Pediatr Rev 1999;20:e71-8.

2. Stege G, Fenton A, Jaffray B. Nihilism in the 1990s: the true mortality of congenital diaphragmatic hernia. Pediatrics 2003;112(3 Pt 1):532-5.

3. Clugston RD, Greer JJ. Diaphragm development and congenital diaphragmatic hernia. Semin Pediatr Surg 2007;16:94-100.

4. Bielinska M, Jay PY, Erlich JM, et al. Molecular genetics of congenital diaphragmatic defects. Ann Med 2007;39:261-74.

5. Gallot D, Marceau G, Coste K, et al. Congenital diaphragmatic hernia: a retinoid-signaling pathway disruption during lung development? Birth Defects Res Part A Clin Mol Teratol 2005;73:523-31.

6. Skari H, Bjornland K, Haugen G, Egeland T, Emblem R. Congenital diaphragmatic hernia: a meta-analysis of mortality factors. J Pediatr Surg 2000;35:1187-97.

7. Jaillard SM, Pierrat V, Dubois A, et al. Outcome at 2 years of infants with congenital diaphragmatic hernia: a population-based study. Ann Thorac Surg 2003;75:250-6.

8. Iocono JA, Cilley RE, Mauger DT, Krummel TM, Dillon PW. Postnatal pulmonary hypertension after repair of congenital diaphragmatic hernia: predicting risk and outcome. J Pediatr Surg 1999;34:349-53.

9. Kamata S, Usui N, Kamiyama M, et al. Long-term follow-up of patients with high-risk congenital diaphragmatic hernia. J Pediatr Surg 2005;40:1833-8.
10. Muratore CS, Kharasch V, Lund DP, et al. Pulmonary morbidity in 100 survivors of congenital diaphragmatic hernia monitored in a multidisciplinary clinic. J Pediatr Surg 2001;36:133-40.

11. Schwartz IP, Bernbaum JC, Rychik J, Grunstein M, D’Agostino J, Polin RA. Pulmonary hypertension in children following extracorporeal membrane oxygenation therapy and repair of congenital diaphragmatic hernia. J Perinatol 1999;19:220-6.

12. Yamataka T, Puri P. Pulmonary artery structural changes in pulmonary hypertension complicating congenital diaphragmatic hernia. J Pediatr Surg 1997;32:387-90.

13. Beals DA, Schloo BL, Vacanti JP, Reid LM, Wilson JM. Pulmonary growth and remodeling in infants with high-risk congenital diaphragmatic hernia. J Pediatr Surg 1992;27:997-1001; discussion 1001-2.

14. Shehata SM, Tibboel D, Sharma HS, Mooi WJ. Impaired structural remodelling of pulmonary arteries in newborns with congenital diaphragmatic hernia: a histological study of 29 cases. J Pathol 1999;189:112-8.

15. Kitagawa M, Hislop A, Boyden EA, Reid L. Lung hypoplasia in congenital diaphragmatic hernia. A quantitative study of airway, artery, and alveolar development. Br J Surg 1971;58:342-6.

16. Reid L. 1976 Edward B.D. Neuhauser lecture: the lung: growth and remodeling in health and disease. AJR Am J Roentgenol 1977;129:777-88.

17. Lally KP, Engle W; American Academy of Pediatrics Section on Surgery; American Academy of Pediatrics Committee on Fetus and Newborn. Postdischarge follow-up of infants with congenital diaphragmatic hernia. Pediatrics 2008;121:627-32.

18. Mourani PM, Sontag MK, Younoszai A, Ivy DD, Abman SH. Clinical utility of echocardiography for the diagnosis and management of pulmonary vascular disease in young children with chronic lung disease. Pediatrics 2008;121:317-25. 